\title{
Inflammatory Cellular Response to Mechanical Ventilation in Elastase-Induced Experimental Emphysema: Role of Preexisting Alveolar Macrophages Infiltration
}

\author{
Anahita Rouze (D), ${ }^{1,2}$ Guillaume Voiriot, ${ }^{1,3}$ Elise Guivarch, ${ }^{1}$ Françoise Roux, ${ }^{1}$ \\ Jeanne Tran Van Nhieu, ${ }^{3,4}$ Daniel Isabey, ${ }^{1,3}$ Laurent Brochard, ${ }^{5}$ Bernard Maitre, ${ }^{1,3,6,7}$ \\ Armand Mekontso-Dessap, ${ }^{1,3,6}$ and Jorge Boczkowski ${ }^{1,3,6}$ \\ ${ }^{1}$ INSERM, Unité U955 (Institut Mondor de Recherche Biomédicale), Créteil, 94010, France \\ ${ }^{2} \mathrm{CHU}$ Lille, Centre de Réanimation, F-59000 Lille, France \\ ${ }^{3}$ Université Paris Est Créteil Val de Marne, Faculté de Médecine, Groupe de Recherche Clinique CARMAS, Créteil, 94010, France \\ ${ }^{4}$ AP-HP, Hôpitaux Universitaires Henri Mondor, Département de Pathologie, Créteil, 94010, France \\ ${ }^{5}$ Interdepartmental Division of Critical Care Medicine, St Michael's Hospital, Toronto, ON, Canada \\ ${ }^{6}$ AP-HP, Hôpitaux Universitaires Henri Mondor, DHU A-TVB, Service de Réanimation Médicale, Créteil, 94010, France \\ ${ }^{7}$ Centre Hospitalier Intercommunal de Créteil, Service de Pneumologie et Pathologie Professionnelle, Créteil, 94010, France
}

Correspondence should be addressed to Anahita Rouze; anahita.rouze@chru-lille.fr

Received 5 July 2018; Revised 7 November 2018; Accepted 11 November 2018; Published 19 December 2018

Academic Editor: Gernot Zissel

Copyright (C) 2018 Anahita Rouze et al. This is an open access article distributed under the Creative Commons Attribution License, which permits unrestricted use, distribution, and reproduction in any medium, provided the original work is properly cited.

\begin{abstract}
An excessive pulmonary inflammatory response could explain the poor prognosis of chronic obstructive pulmonary disease (COPD) patients submitted to invasive mechanical ventilation. The aim of this study was to evaluate the response to normal tidal volume mechanical ventilation in an elastase-induced murine model of pulmonary emphysema. In this model, two time points, associated with different levels of lung inflammation but similar lung destruction, were analyzed. C57BL/6 mice received a tracheal instillation of 5 IU of porcine pancreatic elastase (Elastase mice) or the same volume of saline (Saline mice). Fourteen (D14) and 21 (D21) days after instillation, mice were anesthetized, intubated, and either mechanically ventilated (MV) or maintained on spontaneous ventilation (SV) during two hours. As compared with Saline mice, Elastase mice showed a similarly increased mean chord length and pulmonary compliance at D14 and D21, while bronchoalveolar lavage cellularity was comparable between groups. Lung mechanics was similarly altered during mechanical ventilation in Elastase and Saline mice. Activated alveolar macrophages CD11bmid were present in lung parenchyma in both Elastase SV mice and Elastase MV mice at D14 but were absent at D21 and in Saline mice, indicating an inflammatory state with elastase at D14 only. At D14, Elastase MV mice showed a significant increase in percentage of neutrophils in total lung, as compared with Elastase SV mice. Furthermore, alveolar macrophages of Elastase MV mice at D14 overexpressed Grl, and monocytes showed a trend to overexpression of CD62L, compared with Elastase SV mice. In an elastase-induced model of pulmonary emphysema, normal tidal volume mechanical ventilation may produce an increase in the proportion of pulmonary neutrophils, and an activation of alveolar macrophages and pulmonary monocytes. This response seems to be observed only when the emphysema model shows an underlying inflammation (D14), reflected by the presence of activated alveolar macrophages CD11bmid.
\end{abstract}

\section{Introduction}

Chronic obstructive pulmonary disease (COPD) is characterized by persistent airflow limitation, associated with an excessive inflammatory response to noxious particles or gases in the airways and the lung [1]. Invasive mechanical ventilation may lead to a higher mortality rate in this population [2] and has been recognized as an independent risk factor for mortality among COPD patients admitted to intensive care units (ICU) with acute respiratory failure [3]. 


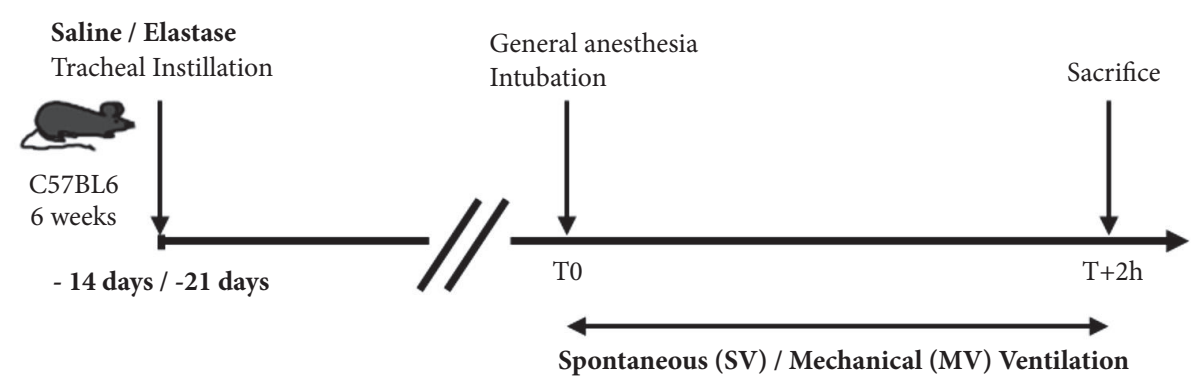

FIGURE 1: Design of experimental groups. C57BL/6 mice received a tracheal instillation of either elastase (Elastase mice) or saline (Saline mice). After 14 or 21 days, mice were anesthetized, intubated, subjected to either spontaneous ventilation (SV mice) or mechanical ventilation (MV mice), and then sacrificed after two hours.

Numerous experimental and clinical studies have reported the concept of ventilator-induced lung injury (VILI) [4-6]. The use of high tidal volumes (Vt) is one of its main contributors, especially leading to an acute inflammation secondary to lung overdistension, and known as "biotrauma" [7-11]. Normal Vt, close to $8 \mathrm{~mL} / \mathrm{kg}$, may also lead to pulmonary inflammation $[10,12,13]$. Furthermore, a preexisting lung inflammatory process could aggravate the inflammatory response to mechanical ventilation [10].

Since chronic airway and lung inflammation play a major role in the pathogenesis of COPD and its alveolar component, emphysema [14-16], we supposed that mechanical ventilation may aggravate preexisting pulmonary inflammation in emphysematous lungs. This phenomenon could explain, at least in part, the detrimental effect of invasive mechanical ventilation in COPD patients. The aim of our study was therefore to evaluate the inflammatory response during two hours of normal Vt mechanical ventilation in a murine model of pulmonary emphysema induced by elastase [17]. This model is characterized by an early and transient alveolar infiltration by macrophages [18-20]. Thereby, in order to examine the effects of preexisting alveolar macrophages infiltration in the inflammatory response to mechanical ventilation, animals were examined at days 14 and 21 after elastase instillation, two time points associated with similar lung destruction, but the presence and the absence of macrophages infiltration, respectively. We hypothesized that mechanical ventilation may induce more pulmonary inflammation, reflected by inflammatory cells influx to lung and activation status of these cells, in D14 as compared to D21 elastase-induced emphysema model.

\section{Methods}

2.1. Animal Model. All the experiments were performed in accordance with the official regulations of the French Ministry of Agriculture and the US National Institute of Health guidelines for the experimental use of animals and were approved by the local institutional animal care and use committee. Six-week-old male C57BL/6 mice (Janvier Labs, Le Genest Saint-Isle, France) received the instillation of either 5 IU of porcine pancreatic elastase (Elastin Products, Owensville, MO, USA) (Elastase mice) or $50 \mu \mathrm{l}$ of saline (Saline mice) into the trachea [20]. Mice were then subjected to subsequent ventilation at two time points, 14 and 21 days after instillation.

2.2. Mechanical Ventilation. Mice were anesthetized and intubated. Mice in mechanical ventilation (MV) group were ventilated for two hours by means of a computerdriven small-animal ventilator (flexiVent, SCIREQ, Montreal, Canada) as follows: $\mathrm{Vt}=8 \mu \mathrm{L} / \mathrm{g}$ of body weight, respiratory rate $=180 / \mathrm{min}$, end-expiratory pressure $=1.5 \mathrm{cmH}_{2} \mathrm{O}$, and fraction of inspired oxygen $=0.4-0.6$ [21]. A control group (SV) consisted of anesthetized, intubated mice, maintained on spontaneous ventilation for two hours.

2.3. Experimental Design. The experimental design included four groups, at two distinct time points from tracheal instillation (D14 and D21) (Figure 1): Saline SV (saline instillation followed by spontaneous ventilation), Elastase SV (elastase instillation followed by spontaneous ventilation), Saline MV (saline instillation followed by mechanical ventilation), and Elastase MV (elastase instillation followed by mechanical ventilation).

2.4. Respiratory Mechanics. The flexiVent ventilator was used for continuous measurement of mean and peak airway pressures (Ppeak, Pmean) and to explore the respiratory mechanics using different waveforms [21]. Single frequency forced oscillation techniques were assessed at initiation of mechanical ventilation, before $(\mathrm{H} 0)$ and after $\left(\mathrm{HO}^{\prime}\right)$ recruitment maneuver, and then repeated hourly ( $\mathrm{H} 1$ and $\mathrm{H} 2$ ), for calculation of respiratory system dynamic resistance and compliance.

2.5. Specimen Collection. After sacrifice, bronchoalveolar lavage (BAL) was performed, lungs were collected for either fixation (4\% paraformaldehyde) and paraffin embedding, or flow cytometric analysis.

2.6. Morphometric Analysis. Sections of $5 \mu \mathrm{m}$ thickness of the left lung were stained with hematoxylin and eosin. Fifteen digital photomicrographs were acquired at x200 magnification in a systematic fashion (Axioplan 2 microscope equipped with an MRc digital color camera (Zeiss, Oberkochen, Germany). Emphysema was quantified by measurement of 
TABLE 1: Respiratory mechanics data during mechanical ventilation in D14 and D21 Saline and Elastase mice.

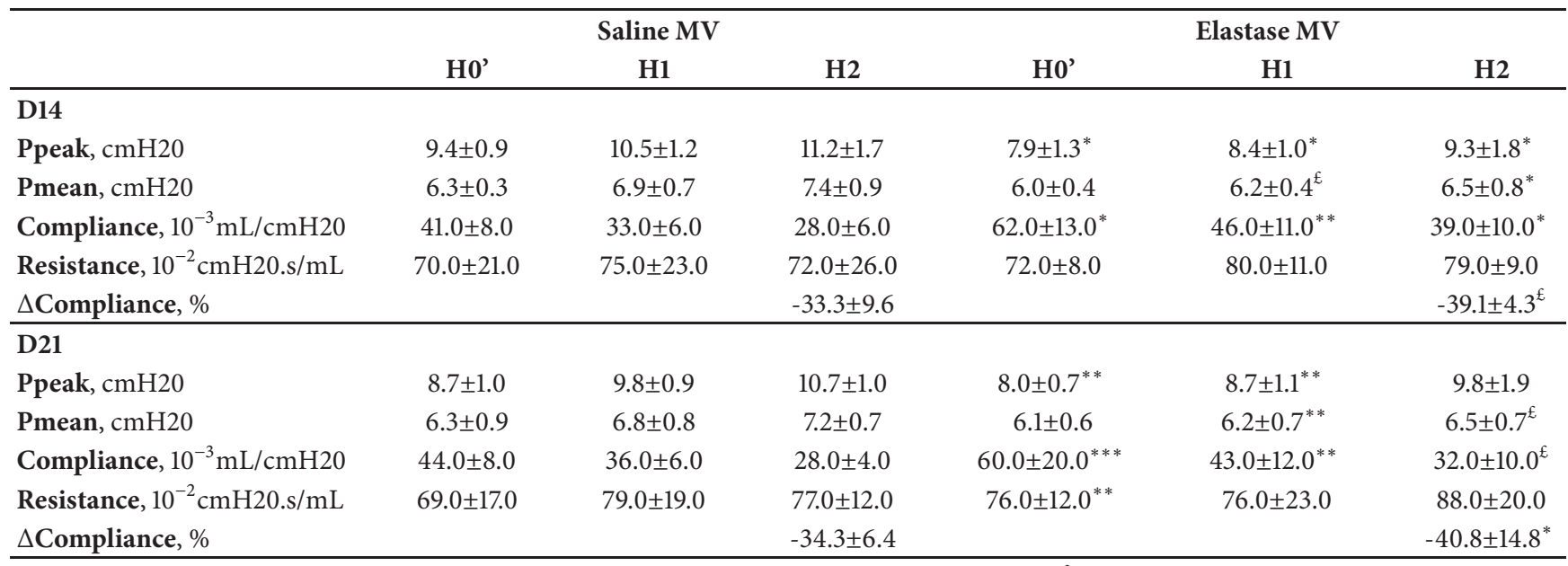

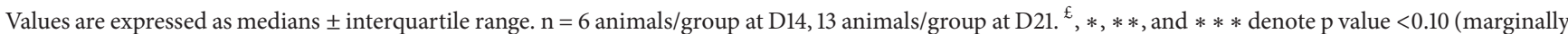
significant), $<0.05,<0.01$, and $<0.001$, respectively, for the Mann-Whitney pairwise comparisons (following Kruskal-Wallis test), Elastase MV versus Saline MV. Definition of abbreviations: MV: mechanical ventilation; $\mathrm{HO}$ ': after volume history standardization consisting of three inflations to a transrespiratory pressure of $30 \mathrm{cmH}_{2} \mathrm{O} ; \mathrm{H1}$ : after one hour; H2: at the end of MV; Ppeak: peak airway pressure; Pmean: mean airway pressure; Compliance, Resistance: dynamic compliance and resistance of the respiratory system calculated using the single frequency forced oscillation technique; $\Delta$ Compliance: percentage of compliance decrease during mechanical ventilation, between $\mathrm{H}^{\prime}$ ' and $\mathrm{H} 2$.

alveolar diameters with an image analysis software (Image), $\mathrm{NIH}$, Bethesda, USA). This automated analysis was made vertically and horizontally on each photomicrograph. The mean chord length of alveoli was obtained by averaging those measurements [20].

2.7. Bronchoalveolar Lavage. The total cell count of BAL was determined using a Malassez hemocytometer (Hycor Biomedical, Indianapolis, IN, USA). Differential cell counts were done on cytocentrifuge preparations (Cytospin 3; Shandon Scientific, Cheshire, UK) stained with Diff-Quick stain (Baxter Diagnostics, McGaw Park, IL, USA).

2.8. Flow Cytometric Analysis. Mechanical disruption followed by enzymatic digestion of murine lungs was performed $[9,22]$. Total lung cell suspensions were obtained, stained with fluorochrome-conjugated anti-mouse antibodies for CD11b, CD11c, Gr1 (Ly6C/G), F4/80, and CD62L (L-selectin) or appropriate isotype-matched controls, and analyzed using a 7-channel cytometer (CyAn ADP Analyzer, Beckman Coulter, Brea, USA) with Summit software (Summit v4.3, Dako, Cambridge, UK). Three inflammatory cell populations in murine lung were identified (see Supplementary Material (available here)). CD11b-, CD11c+ phenotype, with high autofluorescence, defined alveolar macrophages [22]. Monocytes and neutrophils were identified as CD11b+, CD11c-cells but differed especially in their granularity (side-scatter, SS), and F4/80 and Gr1 expression [9, 10]. High SS and F4/80-, Gr1+ phenotype defined neutrophils, whereas low SS, and F4/80+, Grlmid phenotype characterized monocytes. Cells activation state was assessed using expression of CD62L and CD11b adhesion molecule, as well as Grl. All flow cytometric results were presented as relative values, called percentage of gated cells [23].
2.9. Statistical Analysis. Data were analyzed using SPSS Base 16.0 statistical software (SPSS Inc, Chicago, IL, USA). Continuous data were expressed as median \pm interquartile range. Independent samples were compared using KruskalWallis test followed by pairwise Mann-Whitney test, with correction for multiple testing by Benjamini-Hochberg false discovery rate. Two-tailed $\mathrm{p}$ values smaller than 0.10 and 0.05 were considered marginally significant and significant, respectively.

\section{Results}

3.1. Morphometric Analysis (Figure 2). Morphometric analysis showed a marked increase in mean chord length in Elastase mice (at both D14 and D21) as compared to Saline mice.

\subsection{Respiratory Mechanics (Table 1, Figure 3)}

3.2.1. Elastase-Induced Emphysema Model. Elastase mice (at both D14 and D21) exhibited higher values of respiratory system dynamic compliance at the beginning of mechanical ventilation after volume history standardization (recruitment maneuver), as compared to Saline mice.

3.2.2. Effect of Mechanical Ventilation. A gradual decrease in respiratory system compliance (with associated increase in peak airway pressures) was observed in both Elastase MV and Saline MV mice during the two hours of mechanical ventilation. This decrease was similar between Elastase MV and Saline MV mice at D14, but was greater in the former group at D21 $(40.8 \%$ versus $34.3 \%, \mathrm{p}<0.05)$. 


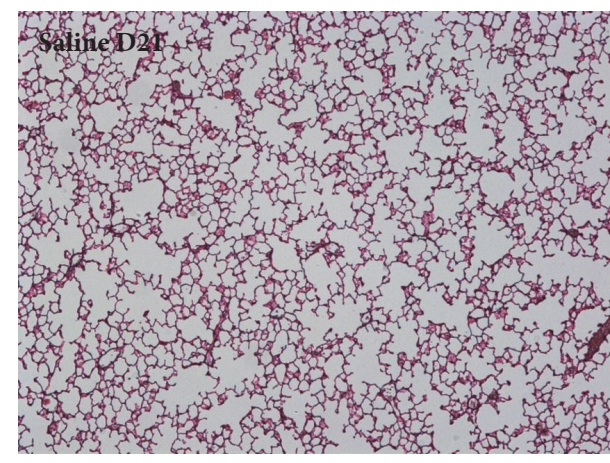

(a)

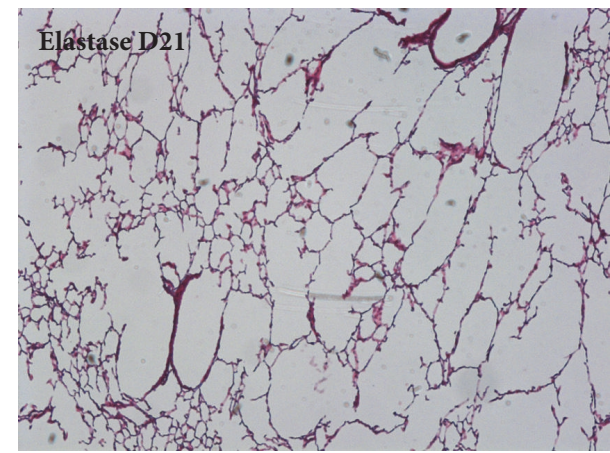

(c)

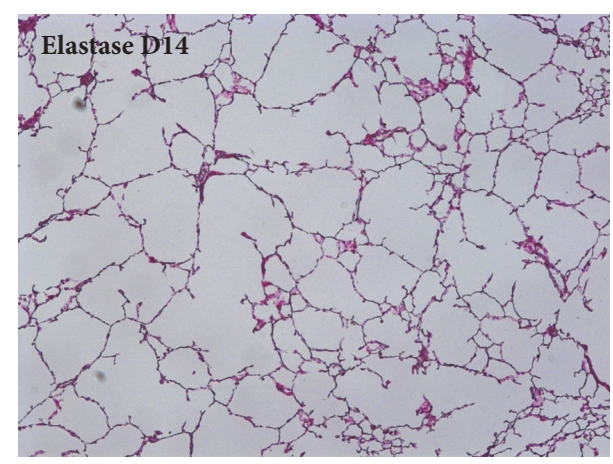

(b)

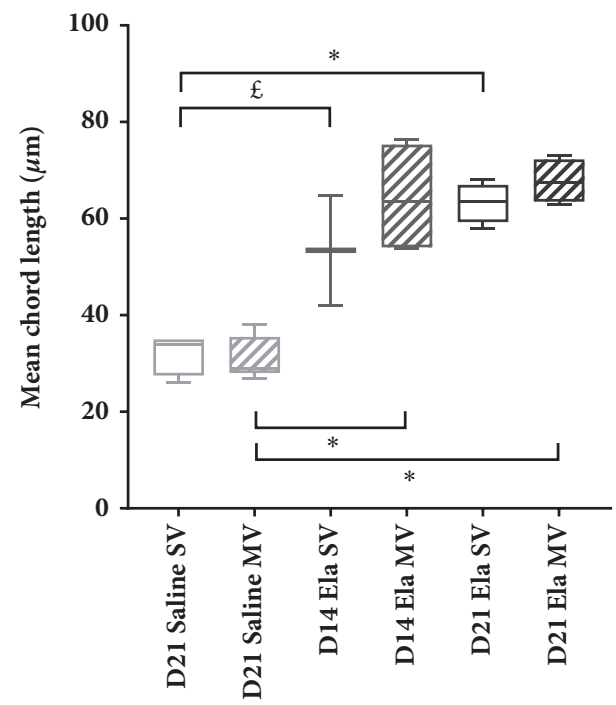

(d)

FIGURE 2: Morphometric analysis. (a), (b), (c) Representative photomicrographs (50-fold magnification) of histological slides of murine lungs. Hematoxylin-eosin staining. Saline SV mice, 21 days after tracheal instillation of saline (a), Elastase SV mice, D14 (b), Elastase SV mice, D21 (c). (d) Mean chord length of alveoli in SV and MV mice, 21 days after saline tracheal instillation, 14 and 21 days after elastase tracheal instillation. Values are expressed as medians \pm interquartile range. $\mathrm{n}=3-5$ animals/group. ${ }^{£}$ and $*$ denote Benjamini-Hochberg corrected $\mathrm{p}$ value $<0.10$ (marginally significant) and $<0.05$, respectively, for the following Mann-Whitney pairwise comparisons (following Kruskal-Wallis test): Saline SV versus Saline MV, Saline SV versus Ela SV D14, Saline SV versus Ela SV D21, Saline MV versus Ela MV D14, Saline MV versus Ela MV D21, Ela SV versus Ela MV D14, Ela SV versus Ela MV D21, Ela SV D14 versus Ela SV D21, Ela MV D14 versus Ela MV D21. Definition of abbreviations: SV: spontaneous ventilation; MV: mechanical ventilation; Ela: elastase tracheal instillation.

\subsection{BAL Cellularity (Table 2)}

3.3.1. Elastase-Induced Emphysema Model. BAL cellularity was similar between Saline and Elastase mice at both D14 and D21, and differential cell count showed a predominance of macrophages ( $>90 \%$ of total cells) in all groups.

3.3.2. Effect of Mechanical Ventilation. BAL cellularity was comparable between Elastase MV, Elastase SV, Saline MV, and Saline SV mice at both D14 and D21 time points.

\subsection{Flow Cytometric Analysis on Total Lung Cell Suspensions (Figures 4-6)}

3.4.1. Elastase-Induced Emphysema Model. Flow cytometric analysis of inflammatory cells from total lung cell suspensions found a marginally significant increase in the percentage of alveolar macrophages in Elastase SV mice compared with Saline SV mice at D14 (Figure 4(a)). These macrophages showed a trend towards an overexpression of CD11b and a stronger autofluorescence, compared with Saline SV mice (Figure 5(a)). Flow cytometric analyses were similar between Elastase SV and Saline SV mice at D21, except for a significant increase in autofluorescence of alveolar macrophages in the former group (Figures 4(b) and 6).

3.4.2. Effect of Mechanical Ventilation. Flow cytometric analysis on total lung cell suspensions did not find any significant change in pulmonary inflammatory cell populations or cellular activation state between Saline MV and Saline SV mice, but with a trend towards increased percentage of neutrophils 


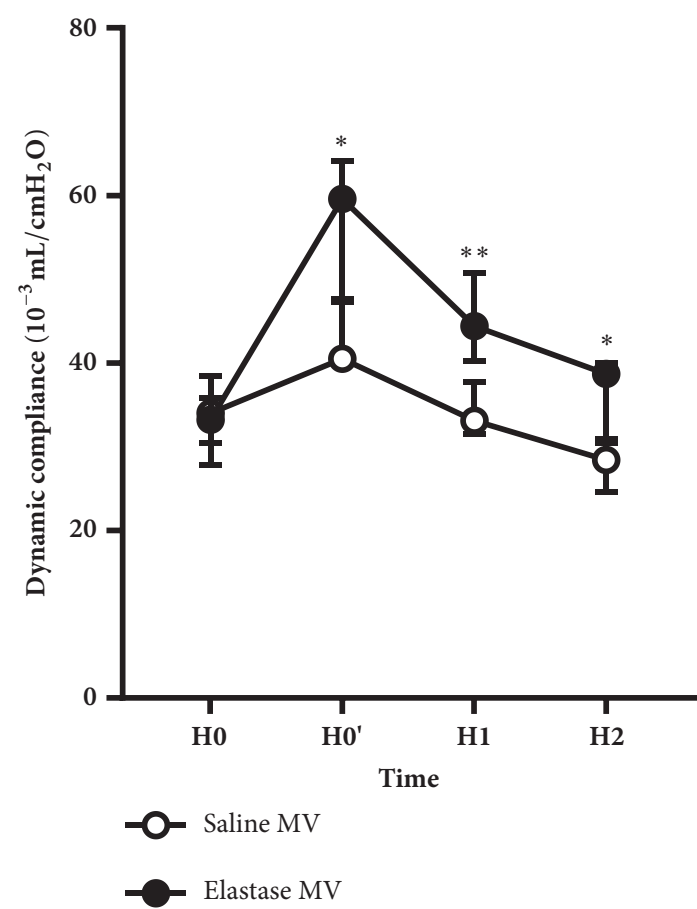

(a) D14

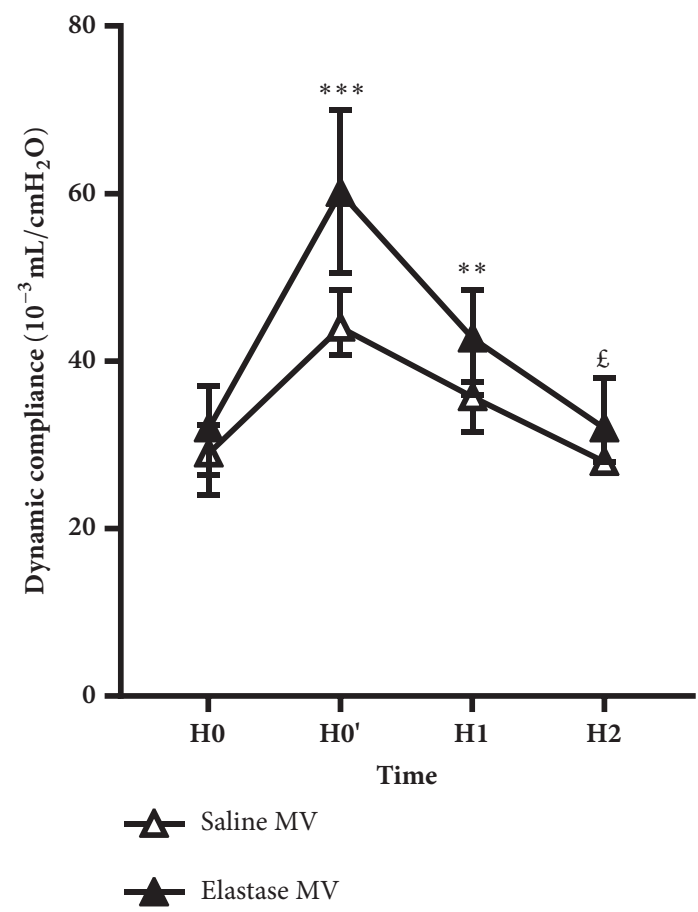

(b) D21

Figure 3: Evolution of dynamic compliance, calculated using the single frequency forced oscillation technique, during two hours of mechanical ventilation in Saline and Elastase mice, at D14 (a) and D21 (b) of tracheal instillation. Values are expressed as median \pm interquartile range. $\mathrm{n}=6$ animals/group at $\mathrm{D} 14$, and 13 animals/group at D21. ${ }^{£}, *, * *$, and $* * *$ denote $\mathrm{p}$ value $<0.10$ (marginally significant), $<0.05$, $<0.01$, and $<0.001$, respectively, for the Mann-Whitney pairwise comparisons (following Kruskal-Wallis test), Elastase MV versus Saline MV. Definition of abbreviations: MV: mechanical ventilation; H0: at ventilator connection; H0': after volume history standardization (recruitment maneuver); H1: after one hour of MV; H2: at the end of MV.

TABLE 2: Bronchoalveolar lavage cellularity in SV and MV mice, 14 and 21 days after saline or elastase tracheal instillation.

\begin{tabular}{|c|c|c|c|c|c|c|c|c|}
\hline & \multicolumn{4}{|c|}{ D14 } & \multicolumn{4}{|c|}{ D21 } \\
\hline & \multicolumn{2}{|c|}{ Saline } & \multicolumn{2}{|c|}{ Elastase } & \multicolumn{2}{|c|}{ Saline } & \multicolumn{2}{|c|}{ Elastase } \\
\hline & SV & MV & SV & MV & SV & MV & SV & MV \\
\hline Cell count, $\times 10^{4}$ cell $/ \mu \mathrm{L}$ & $8.4 \pm 8.7$ & $11.5 \pm 6.5$ & $8.2 \pm 10.6$ & $9.3 \pm 8.8$ & $9.1 \pm 4.4$ & $11.1 \pm 11.3$ & $11.2 \pm 4.9$ & $11.3 \pm 6.8$ \\
\hline
\end{tabular}

Definition of abbreviations: SV: spontaneous ventilation; MV: mechanical ventilation.

Values are expressed as median \pm interquartile range; $n=5-9$ animals/group.

No significant difference was found between groups. Differential cell count showed a predominance of macrophages ( $>90 \%$ of total cells) in all groups.

and increased CD62L expression on monocytes in the former group (Figures 4 and 5(b)).

A significant decrease in the percentage of alveolar macrophages (with a concomitant increase in the percentage of neutrophils) was noted at D14 in Elastase MV mice as compared to Elastase SV mice (Figure 4(a)). This was associated with a change in alveolar macrophages phenotype, with a marginally significant overexpression of Gr1 in the former group (Figure 5(a)). Pulmonary monocytes also exhibited a modified phenotype, with a maximal expression of CD62L in Elastase MV mice (Figure 5(b)). A gradual increase in CD62L expression on monocytes was observed, in relation to the successive assaults between Saline SV, Elastase SV, Saline MV, and Elastase MV groups. All the above-mentioned flow cytometry differences were not observed at D21 time point, except for a stronger autofluorescence of alveolar macrophages in Elastase MV mice as compared to Saline MV mice, which was present both at D14 and D21 (Figures 4(b) and 6).

\section{Discussion}

Main results of our study are as follows: (i) elastase instillation resulted in a similarly increased mean chord length and respiratory system compliance at D14 and D21, as compared to saline instillation; (ii) these alterations were associated with a transient lung infiltration (only at D14) of activated alveolar macrophages CD11bmid; (iii) lung mechanics was similarly altered during a two-hour mechanical ventilation in Elastase and Saline mice, with a gradual decrease in respiratory system compliance; (iv) at D14, mechanically ventilated Elastase mice showed a significant increase in the percentage of 

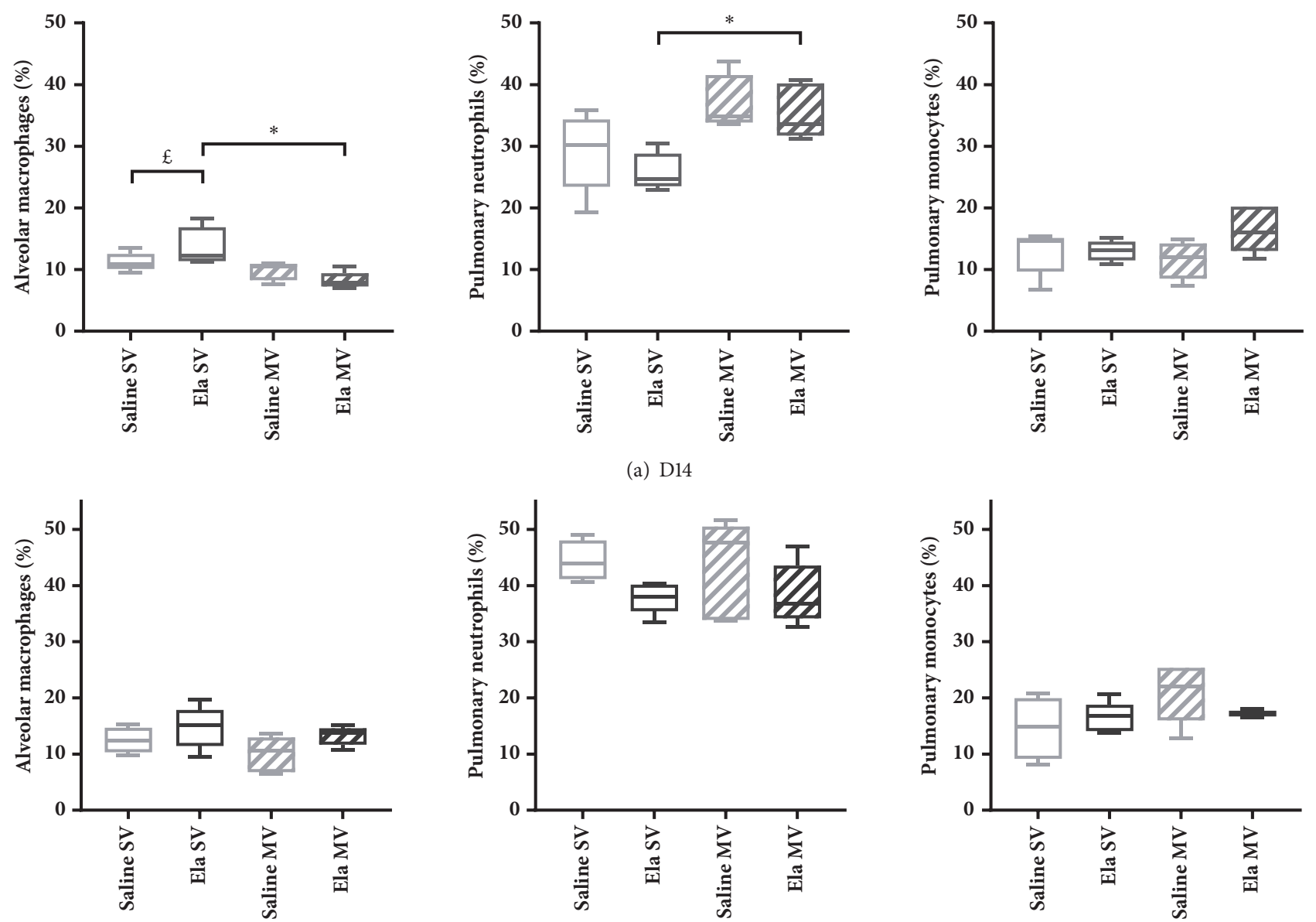

(b) D21

FIGURE 4: Relative values of pulmonary inflammatory cell populations, alveolar macrophages, pulmonary neutrophils, and pulmonary monocytes, analyzed by flow cytometry on total lung cell suspensions of mice subjected to spontaneous (SV) or mechanical (MV) ventilation 14 days (a) and 21 days (b) after instillation of saline (Saline) or elastase (Ela), expressed as percentage of gated cells. Values are expressed as median \pm interquartile range. $\mathrm{n}=5$ animals/group. ${ }^{£}$ and $*$ denote Benjamini-Hochberg corrected $\mathrm{p}$ value $<0.10$ (marginally significant) and $<0.05$, respectively, for the following Mann-Whitney pairwise comparisons (following Kruskal-Wallis test): Saline SV versus Saline MV, Saline SV versus Ela SV, Ela SV versus Ela MV, Saline MV versus Ela MV. Definition of abbreviations: Ela: elastase; SV: spontaneous ventilation; MV: mechanical ventilation.

neutrophils concomitant with a decrease in the percentage of alveolar macrophages in total lung, compared with Elastase animals spontaneously ventilated. Furthermore, alveolar macrophages of mechanically ventilated Elastase mice at D14 overexpressed Grl, whereas monocytes showed a trend to overexpression of CD62L.

Elastase-induced emphysema model has been described in numerous studies [17-20, 24]. Murine lungs undergo an intense inflammatory reaction within the first week after elastase instillation, which results in release of reactive oxygen species and proteases, matrix degradation, and also death of structural cells, and then show a minimal inflammation state in the late phase, after D21, when lung tissue is altered and deserted by inflammatory cells. D14 and D21, being focused on, were allowed to subtly modulate basal inflammation level of our emphysema model, while keeping the same degree of histological emphysema and similar mechanical properties. Many clinical phenotypes of COPD patients are related to the intensity of baseline pulmonary inflammation, such as alveolar destruction, dynamic hyperinflation, exacerbations, or overall prognosis. We believe that this dual model may closely reflect different inflammation patterns found in human emphysematous lungs [14, 25]. Moreover, we used a dose of elastase inducing a degree of emphysema similar to that observed after cigarette smoke exposure (near 30\% increase in mean chord length) [26]. Time course of histological emphysema and alveolar macrophages infiltration observed only at D14 in Elastase SV animals is consistent with previous data from the literature [18-20, 24]. To our knowledge, our study is the first to provide phenotypic characterization of alveolar macrophages in elastase model, by showing overexpression of CD11b in these cells at D14. These macrophages are similar in their phenotype to pulmonary interstitial macrophages [22, 27], but their strong autofluorescence identifies them as alveolar macrophages. $\mathrm{CD} 11 \mathrm{~b}$ is an adhesion molecule whose expression reflects the 

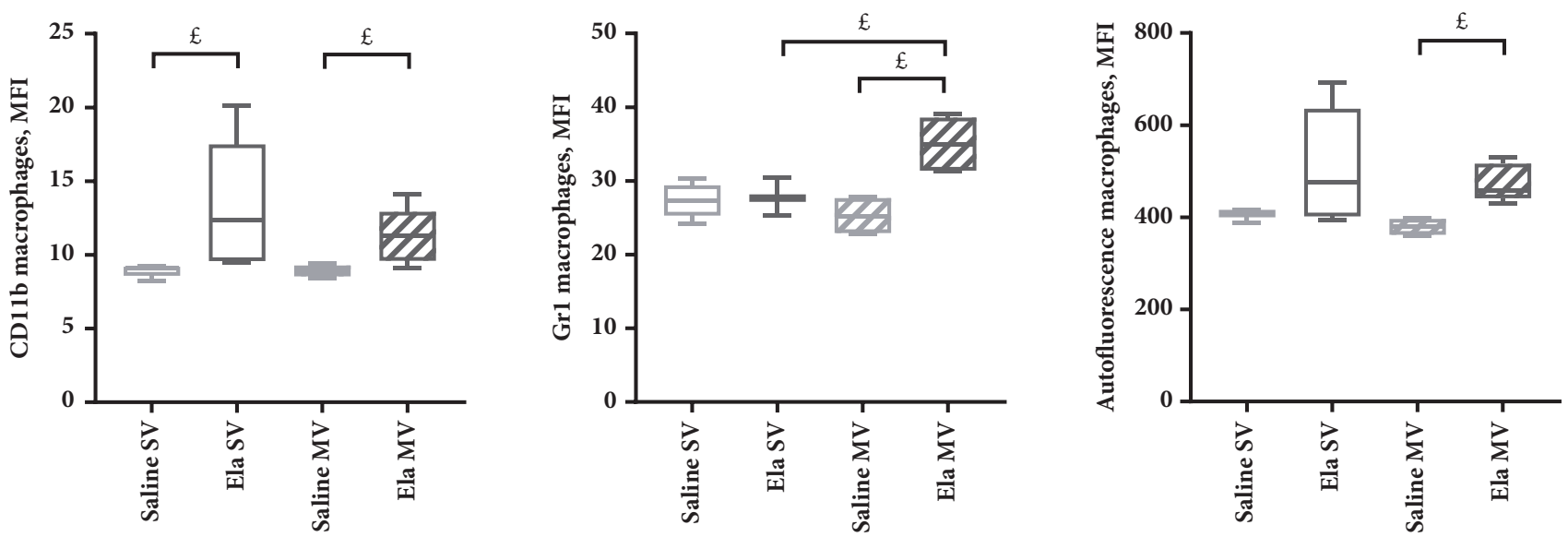

(a) Alveolar macrophages
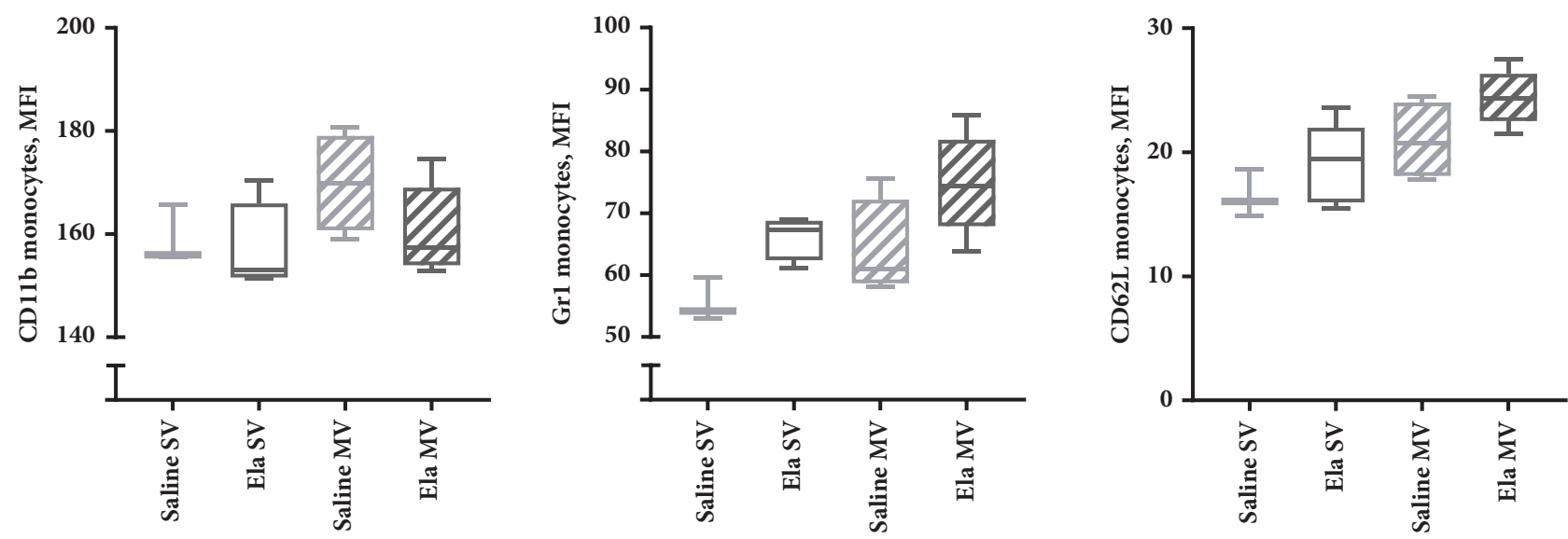

(b) Pulmonary monocytes

Figure 5: Activation markers of alveolar macrophages (a) and pulmonary monocytes (b), analyzed by flow cytometry on total lung cell suspensions of mice subjected to spontaneous (SV) or mechanical (MV) ventilation 14 days after instillation of saline (Saline) or elastase (Ela), expressed as mean fluorescent intensity (MFI). (a) CD11b and Grl expression, and autofluorescence of alveolar macrophages. (b) CD11b, Gr1, and CD62L expression on pulmonary monocytes. Values are expressed as median \pm interquartile range. $\mathrm{n}=3-5$ animals/group. ${ }^{\mathfrak{E}}$ denotes Benjamini-Hochberg corrected $\mathrm{p}$ value $<0.10$ (marginally significant) for the following Mann-Whitney pairwise comparisons (following Kruskal-Wallis test): Saline SV versus Saline MV, Saline SV versus Ela SV, Ela SV versus Ela MV, Saline MV versus Ela MV. Definition of abbreviations: Ela: elastase; SV: spontaneous ventilation; MV: mechanical ventilation.

level of activation of various inflammatory cells. Activated alveolar macrophages play a central role in the pathophysiology of pulmonary emphysema in mice and humans [28]. An increased expression of CD1lb has been reported on macrophages collected in induced sputum of COPD patients. Interestingly, CD11b expression intensity was correlated with the severity of airflow limitation [29]. High autofluorescence of alveolar macrophages, as observed in Elastase mice (in either SV or MV groups, at both instillation times), has been previously reported in the BAL of smokers [30]. A link with tobacco particles phagocytosis has been suggested, without clear biological significance.

We chose normal Vt strategy for its clinical relevance, as Vt close to $8 \mathrm{ml} / \mathrm{kg}$ are now widespread in most mechanically ventilated patients [31]. In terms of respiratory mechanics, both D14 and D21 Elastase mice responded to mechanical ventilation similarly to Saline mice, decreasing to the same extent their respiratory system compliance within two hours of ventilation. This decrease is reported in various ventilated murine models and results from progressive alveolar derecruitment [32]. An identical mechanical response to normal Vt mechanical ventilation has been observed in a TIMP3 KO murine model of emphysema [33]. Following two hours of normal Vt mechanical ventilation, we observed a significant increase in the percentage of neutrophils concomitant with decrease in the percentage of alveolar macrophages only in D14 Elastase mice. Neutrophils recruitment to the lung has been already reported as an important mechanism in VILI [9]. Cell phenotype changes included Grl overexpression by alveolar macrophages and CD62L overexpression by pulmonary monocytes. Grl overexpression is the witness of an activation of macrophages and has already been demonstrated in infectious circumstances [34]. Besides, CD62L is an adhesion molecule whose overexpression on pulmonary 

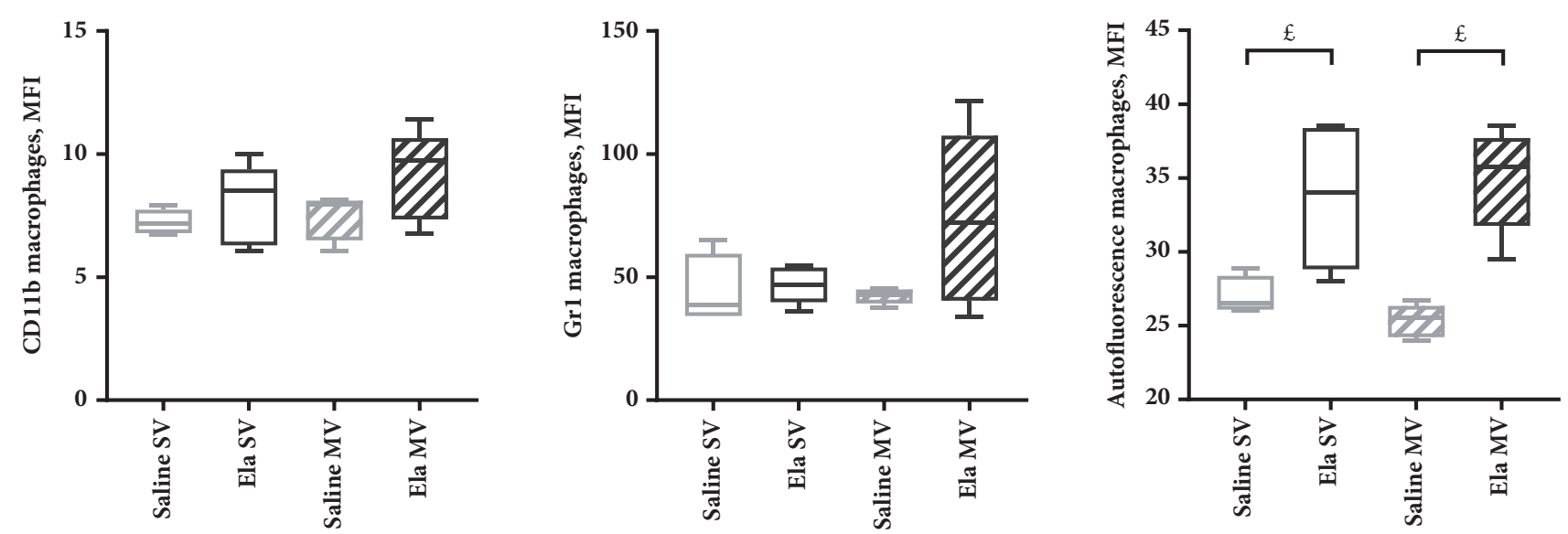

FIGURE 6: CD11b and Grl expression, and autofluorescence of alveolar macrophages, analyzed by flow cytometry on total lung cell suspensions of mice subjected to spontaneous (SV) or mechanical (MV) ventilation 21 days after instillation of saline (Saline) or elastase (Ela), expressed as mean fluorescent intensity (MFI). Values are expressed as median \pm interquartile range. $n=5$ animals/group. ${ }^{\mathfrak{E}}$ denotes Benjamini-Hochberg corrected $\mathrm{p}$ value $<0.10$ (marginally significant) for the following Mann-Whitney pairwise comparisons (following Kruskal-Wallis test): Saline SV versus Saline MV, Saline SV versus Ela SV, Ela SV versus Ela MV, Saline MV versus Ela MV. Definition of abbreviations: Ela: elastase; SV: spontaneous ventilation; MV: mechanical ventilation.

monocytes has already been observed during mechanical ventilation and explained by cellular activation related to stretch [10]. We postulate that inflammatory response to mechanical ventilation in Elastase mice could be related to preexisting inflammation reflected by the presence of activated alveolar macrophages CD11bmid, and not to altered cellular mechanical properties secondary to parenchymal destruction. Indeed, although morphological and functional lung modifications were similar in Elastase mice at D14 and D21 as compared to Saline animals, no modification in both proportions and activation state of pulmonary inflammatory cells was seen in Elastase mice at D21. Previous data have already demonstrated the early and central role of activation of alveolar macrophages subjected to stretch in the initiation of the inflammatory response to mechanical ventilation [11]. Preexisting macrophage activation could predispose these cells to further activation by mechanical ventilation. Whatever the underlining molecular mechanism, this pulmonary inflammatory response following mechanical ventilation could play a deleterious role in the progression of pulmonary emphysema, through a worsening of lung inflammation level $[25,28]$.

Our study had some limitations. First, only male C57BL/6 mice were used to perform all the experimental procedures, as usual in both elastase-induced emphysema model and protocol of mechanical ventilation of mice under general anesthesia commonly performed by our team $[20,21]$. Indeed, female mice appear more susceptible to distress, arrhythmias, and cardiac arrest than male mouse in response to various anesthesia procedures $[35,36]$. As a result, our data should be interpreted with caution, taking into account this sex bias. Further, morphometric data were not obtained for the saline group at day 14, and results from D21 mice were extrapolated to the D14 group. Besides, a complex calibration protocol was led for each set of flow cytometric analysis. This calibration was valid for a given day analysis, and no comparison could be made between experiments carried out on different days, since the reported variations would not be related to differences between groups but differences in cytometer calibration. Thanks to the limited number of samples simultaneously analyzed, we were not able to directly compare the data from D14 and D21 mice. Besides, our cytometer did not allow automated simultaneous counting of collected lung cells, resulting in inaccurate absolute values [23]. Thus, all flow cytometric results were presented as relative values. As a result, lung recruitment mechanisms could only be suspected, and their existence remains to be confirmed by other techniques allowing absolute values quantification. Nevertheless, the activation of inflammatory cells, through change of cell surface molecule expression, could be more directly interpreted. Our data may be interpreted cautiously as regards technical limits pointed out above, and our conclusions need to be confirmed by crossing with other markers of inflammation. It is worthy of note that we did not identify any substantial variations in inflammatory cell populations in total lung in Saline mice after normal Vt mechanical ventilation, unlike a previous study with a very close experimental protocol, which highlighted increased number of neutrophils in total lung and increased expression of CD62L on pulmonary monocytes, in response to normal Vt mechanical ventilation in healthy mice [10]. However, we observed a trend towards an increased percentage of neutrophils and expression of CD62L on lung monocytes of Saline MV mice as compared to Saline SV mice. The nature of our control group may explain this lack of significance. Indeed, the Saline SV group (consisting of mice instilled with saline, anesthetized, intubated, and maintained in spontaneous ventilation for two hours) was probably subjected to some level of aggression, including pulmonary microatelectasis due to hypoventilation. Finally, we did not use an infectious challenge (e.g., lipopolysaccharide instillation) in conjunction with elastase instillation 
and mechanical ventilation. Such a triple hit model may be closer to the frequent clinical scenario of COPD patients requiring mechanical ventilation because of pneumonia, but its implementation and interpretation may be complex.

\section{Conclusion}

In an elastase-induced model of pulmonary emphysema, normal tidal volume mechanical ventilation may produce an increase in the proportion of pulmonary neutrophils and activation of alveolar macrophages and pulmonary monocytes. This response seems to be observed only when the emphysema model shows an underlying inflammation (D14), reflected by the presence of activated alveolar macrophages CD11bmid.

\section{Data Availability}

The data used to support the findings of this study are available from the corresponding author upon request.

\section{Disclosure}

Some of our results have been previously presented as an abstract (Am J Respir Crit Care Med 2012; 185:A5437).

\section{Conflicts of Interest}

The authors declare that there are no conflicts of interest regarding the publication of this paper.

\section{Authors' Contributions}

Study concept and design were contributed by Anahita Rouze, Guillaume Voiriot, Elise Guivarch, Françoise Roux, Daniel Isabey, Laurent Brochard, Bernard Maitre, Armand Mekontso-Dessap, and Jorge Boczkowski. Acquisition of data was made by Anahita Rouze, Guillaume Voiriot, Elise Guivarch, Françoise Roux, and Jeanne Tran Van Nhieu. Analysis and interpretation of data were performed by Anahita Rouze, Guillaume Voiriot, Elise Guivarch, Françoise Roux, Jeanne Tran Van Nhieu, Bernard Maitre, Armand MekontsoDessap, and Jorge Boczkowski. Draft of the manuscript was prepared by Anahita Rouze, Guillaume Voiriot, Elise Guivarch, Armand Mekontso-Dessap, and Jorge Boczkowski. Statistical analysis was carried out by Anahita Rouze, Guillaume Voiriot, and Armand Mekontso-Dessap. Guillaume Voiriot and Jorge Boczkowski were responsible for study supervision. All authors critically reviewed the manuscript for important intellectual content and read and approved the final manuscript.

\section{Acknowledgments}

We are very grateful to the following members of U955 INSERM Unit for their scientific and technical assistance: Stéphane Kerbrat, Dr. Sabine Le Gouvello, Dr. Laurent
Boyer (Equipe 4, INSERM U955, Créteil), Rachid Souktani (Plateforme Exploration Fonctionnelle du Petit Animal, INSERM U955, Créteil), Aurélie Guguin and Adeline Henri (Plateforme Cytométrie en Flux, INSERM U955, Créteil), and Xavier Decrouy (Plateforme Imagerie, INSERM U955, Créteil).

\section{Supplementary Materials}

Additional information about the methods are available in supplementary material file, including arterial blood gases data, fluorochrome-conjugated anti-mouse antibodies used, and flow cytometric analysis of inflammatory cell populations in mouse lungs. (Supplementary Materials)

\section{References}

[1] J. Vestbo, S. S. Hurd, A. G. Agustí et al., "Global strategy for the diagnosis, management, and prevention of chronic obstructive pulmonary disease: GOLD executive summary," American Journal of Respiratory and Critical Care Medicine, vol. 187, no. 4, pp. 347-365, 2013.

[2] N. Molinari, C. Briand, I. Vachier et al., "Hospitalizations for COPD exacerbations: Trends and determinants of death," CopdJournal of Chronic Obstructive Pulmonary Disease, vol. 12, no. 6, pp. 621-627, 2015.

[3] E. Akkutuk, Z. Karakurt, C. Salturk et al., "How do COPD comorbidities affect ICU outcomes?" International Journal of Chronic Obstructive Pulmonary Disease, p. 1187, 2014.

[4] J. R. Beitler, A. Malhotra, and B. T. Thompson, "Ventilatorinduced Lung Injury," Clinics in Chest Medicine, vol. 37, no. 4, pp. 633-646, 2016.

[5] R. G. Brower, M. A. Matthay, A. Morris, D. Schoenfeld, B. T. Thompson, and A. Wheeler, "Ventilation with lower tidal volumes as compared with traditional tidal volumes for acute lung injury and the acute respiratory distress syndrome," The New England Journal of Medicine, vol. 342, no. 18, pp. 1301-1308, 2000.

[6] A. Malhotra, "Low-tidal-volume ventilation in the acute respiratory distress syndrome," The New England Journal of Medicine, vol. 357, no. 11, pp. 1113-1120, 2007.

[7] F. J. J. Halbertsma, M. Vaneker, G. J. Scheffer, and J. G. van der Hoeven, "Cytokines and biotrauma in ventilator-induced lung injury: A critical review of the literature," The Netherlands Journal of Medicine, vol. 63, no. 10, pp. 382-392, 2005.

[8] J. A. Belperio, M. P. Keane, M. D. Burdick et al., "Critical role for CXCR2 and CXCR2 ligands during the pathogenesis of ventilator-induced lung injury," The Journal of Clinical Investigation, vol. 110, no. 11, pp. 1703-1716, 2002.

[9] S. Choudhury, M. R. Wilson, M. E. Goddard, K. P. O’Dea, and M. Takata, "Mechanisms of early pulmonary neutrophil sequestration in ventilator-induced lung injury in mice," American Journal of Physiology-Lung Cellular and Molecular Physiology, vol. 287, no. 5, pp. L902-L910, 2004.

[10] M. R. Wilson, K. P. O’Dea, D. Zhang, A. D. Shearman, N. Van Rooijen, and M. Takata, "Role of lung-marginated monocytes in an in vivo mouse model of ventilator-induced lung injury," American Journal of Respiratory and Critical Care Medicine, vol. 179, no. 10, pp. 914-922, 2009. 
[11] J. A. Frank, C. M. Wray, D. F. McAuley, R. Schwendener, and M. A. Matthay, "Alveolar macrophages contribute to alveolar barrier dysfunction in ventilator-induced lung injury," American Journal of Physiology-Lung Cellular and Molecular Physiology, vol. 291, no. 6, pp. L1191-L1198, 2006.

[12] E. K. Wolthuis, A. P. J. Vlaar, G. Choi, J. J. T. H. Roelofs, N. P. Juffermans, and M. J. Schultz, "Mechanical ventilation using non-injurious ventilation settings causes lung injury in the absence of pre-existing lung injury in healthy mice.," Critical Care (London, England), vol. 13, no. 1, p. R1, 2009.

[13] K. Vaporidi, G. Voloudakis, G. Priniannakis et al., "Effects of respiratory rate on ventilator-induced lung injury at a constant Paco2 in a mouse model of normal lung," Critical Care Medicine, vol. 36, no. 4, pp. 1277-1283, 2008.

[14] T. Yoshida and R. M. Tuder, "Pathobiology of cigarette smokeinduced chronic obstructive pulmonary disease," Physiological Reviews, vol. 87, no. 3, pp. 1047-1082, 2007.

[15] R. T. Abboud and S. Vimalanathan, "Pathogenesis of COPD. Part I. The role of protease-antiprotease imbalance in emphysema," International Journal of Tuberculosis and Lung Disease, vol. 12, no. 4, p. 3617, 2008.

[16] N. Angelis, K. Porpodis, P. Zarogoulidis, D. Spyratos, I. Kioumis, and A. Papaiwannou, "Airway inflammation in chronic obstructive pulmonary disease," Journal of Thoracic Disease, vol. 6, 1, 2014.

[17] B. Suki, E. Bartolák-Suki, and P. R. M. Rocco, "Elastase-induced lung emphysema models in mice," Methods in Molecular Biology, vol. 1639, pp. 67-75, 2017.

[18] A. M. Houghton, P. A. Quintero, D. L. Perkins et al., "Elastin fragments drive disease progression in a murine model of emphysema," The Journal of Clinical Investigation, vol. 116, no. 3, pp. 753-759, 2006.

[19] S. Tasaka, K.-I. Inoue, K. Miyamoto et al., "Role of interleukin6 in elastase-induced lung inflammatory changes in mice," Experimental Lung Research, vol. 36, no. 6, pp. 362-372, 2010.

[20] L. Plantier, S. Marchand-Adam, V. G. Antico et al., "Keratinocyte growth factor protects against elastase-induced pulmonary emphysema in mice," American Journal of PhysiologyLung Cellular and Molecular Physiology, vol. 293, no. 5, pp. L1230-L1239, 2007.

[21] A. M. Dessap, G. Voiriot, T. Zhou et al., "Conflicting physiological and genomic cardiopulmonary effects of recruitment maneuvers in murine acute lung injury," American Journal of Respiratory Cell and Molecular Biology, vol. 46, no. 4, pp. 541550, 2012.

[22] M. Gonzalez-Juarrero, T. S. Shim, A. Kipnis, A. P. JunqueiraKipnis, and I. M. Orme, "Dynamics of macrophage cell populations during murine pulmonary tuberculosis," The Journal of Immunology, vol. 171, no. 6, pp. 3128-3135, 2003.

[23] B. Brando, D. Barnett, G. Janossy et al., "Cytofluorometric methods for assessing absolute numbers of cell subsets in blood," Cytometry, vol. 42, no. 6, pp. 327-346, 2000.

[24] M. Mouded, E. E. Egea, M. J. Brown et al., "Epithelial cell apoptosis causes acute lung injury masquerading as emphysema," American Journal of Respiratory Cell and Molecular Biology, vol. 41, no. 4, pp. 407-414, 2009.

[25] T. Perez, H. Mal, B. Aguilaniu, P-Y. Brillet, A. Chaouat, and R. Louis, "[COPD and inflammation: statement from a French expert group. Phenotypes related to inflammation]," Revue des Maladies Respiratoires, vol. 28, no. 2, Article ID 15, 2011.

[26] J. L. Wright, M. Cosio, and A. Churg, "Animal models of chronic obstructive pulmonary disease," American Journal of
Physiology-Lung Cellular and Molecular Physiology, vol. 295, no. 1, pp. L1-L15, 2008.

[27] Y. Aida, Y. Shibata, S. Abe et al., "Inhibition of elastasepulmonary emphysema in dominant-negative MafB transgenic mice," International Journal of Biological Sciences, vol. 10, no. 8, pp. 882-894, 2014.

[28] P. G. Woodruff, L. L. Koth, Y. H. Yang et al., "A distinctive alveolar macrophage activation state induced by cigarette smoking," American Journal of Respiratory and Critical Care Medicine, vol. 172, no. 11, pp. 1383-1392, 2005.

[29] J. Domagala-Kulawik, M. Maskey-Warzechowska, J. Hermanowicz-Salamon, and R. Chazan, "Expression of macrophage surface markers in induced sputum of patients with chronic obstructive pulmonary disease," Journal of Physiology and Pharmacology, p. 57, 2006.

[30] C. M. Sköld, A. Eklund, G. Halldén, and J. Hed, "Autofluorescence in human alveolar macrophages from smokers: Relation to cell surface markers and phagocytosis," Experimental Lung Research, vol. 15, no. 6, pp. 823-835, 1989.

[31] O. Gajic, S. I. Dara, J. L. Mendez et al., "Ventilator-associated lung injury in patients without acute lung injury at the onset of mechanical ventilation," Critical Care Medicine, vol. 32, no. 9, pp. 1817-1824, 2004.

[32] G. B. Allen, B. T. Suratt, L. Rinaldi, J. M. Petty, and J. H. Bates, "Choosing the frequency of deep inflation in mice: balancing recruitment against ventilator-induced lung injury," American Journal of Physiology-Lung Cellular and Molecular Physiology, vol. 291, no. 4, pp. L710-L717, 2006.

[33] E. L. Martin, E. A. Truscott, T. C. Bailey et al., "Lung mechanics in the TIMP3 null mouse and its response to mechanical ventilation," Experimental Lung Research, vol. 33, no. 2, pp. 99113, 2007.

[34] D. G. Mordue and L. D. Sibley, "A novel population of Gr-1 activated macrophages induced during acute toxoplasmosis," Journal of Leukocyte Biology, vol. 74, no. 6, pp. 1015-1025, 2003.

[35] M.-D. Drici, L. Baker, P. Plan, J. Barhanin, G. Romey, and G. Salama, "Mice display sex differences in halothane-induced polymorphic ventricular tachycardia," Circulation, vol. 106, no. 4, pp. 497-503, 2002.

[36] K. Hohlbaum, B. Bert, S. Dietze, R. Palme, H. Fink, and C. Thöne-Reineke, "Severity classification of repeated isoflurane anesthesia in C57BL/6JRj mice - Assessing the degree of distress," PLoS ONE, vol. 12, no. 6, 2017. 